\title{
Magnetoresistance of microstructured permalloy ellipses having multi-domain configurations
}

\author{
C.Y. Kuo ${ }^{\text {a }}$, W.S. Chung ${ }^{\mathrm{a}}$, J.C. Wu ${ }^{\mathrm{a}, *}$, Lance Horng ${ }^{\mathrm{a}}$, Zung-Hang Wei ${ }^{\mathrm{b}}$, \\ Mei-Feng Laic, Ching-Ray Chang ${ }^{\mathrm{d}}$ \\ ${ }^{a}$ Taiwan SPIN Research Center and Department of Physics, National Changhua University of Education, Changhua 500, Taiwan \\ ${ }^{\mathrm{b}}$ Department of Power Mechanical Engineering, National Tsing Hua University, HsinChu 300, Taiwan, ROC \\ ${ }^{c}$ Department of Engineering Science and Ocean Engineering, National Taiwan University, Taipei 10607, Taiwan, ROC \\ ${ }^{\mathrm{d}}$ Center for Nanostorage Research, Department of Physics, National Taiwan University, Taipei 10607, Taiwan, ROC
}

Available online 27 November 2006

\begin{abstract}
Mirostructured permalloy ellipses having purposely designed multi-domain configurations were investigated. The samples were fabricated using e-beam lithography through a lift-off process. The magnetoresistance measurements were carried out with a constant dc sensing current under the external magnetic field applied along the short axis. The magnetoresistance curves manifest characteristic features in accordance with the specific domain configurations. Step-like/kink features were observed on the ellipses with cross-tie wall/ two-vortex configuration and step-like plus kink magnetorsistance curve was found on the ellipse with cross-tie wall combining with twovortex structure. A magnetic force microscopy and a micromagnetic simulation were employed to support these results.
\end{abstract}

(C) 2006 Elsevier B.V. All rights reserved.

PACS: 75.60.-d; 70.50.Cc

Keywords: Cross-tie wall; Vortex; Magnetic-force microscope (MFM); Magnetoresistance (MR); Permalloy

Over the years, patterned magnetic thin films have been studied extensively mainly due to potential applications for magnetic field sensors and magnetic data storage and memory [1-3]. Among many efforts leading towards applications, the electrical characteristics of free layer, which permalloy thin film is commonly used, with various shapes and dimensions need to be thoroughly understood. In the various examples demonstrated in the past the magnetic elements were focused on elliptical shapes mainly because of its switching robustness [4]. However, the domain structures may inevitably exist in the elliptical elements causing more electron-scattering from the domain walls. Consequently, the magnetoresistance (MR) curve can represent the effects resulted from domain walls contribution [5]. Herein, we present a study exploring the unique MR curves measured on the purposely designed elliptical permalloy elements. The main theme was to look

\footnotetext{
*Corresponding author. Tel.: + 886047253896 ; fax: + 886047253896 .

E-mail address: phjcwu@cc.ncue.edu.tw (J.C. Wu).
}

into the characteristics of MR curves of the permalloy ellipses having various magnetizations, such as uniform magnetization, cross-tie walls, vortex configurations, and vortex combined with cross-tie walls.

The dimensions of the ellipses are as follows. Sample A has long/short axis of $2 / 1 \mu \mathrm{m}$ and film thickness of $40 \mathrm{~nm}$. Sample B has long/short axis of $8 / 1 \mu \mathrm{m}$ and film thickness of $55 \mathrm{~nm}$. Sample $C$ has long/short axis of $5 / 1 \mu \mathrm{m}$ and film thickness of $33 \mathrm{~nm}$. These ellipses were fabricated using two-step standard electron beam lithography and thermal evaporation. First, a Hitachi S3000 H scanning electron microscope modified for direct writing was employed for creating the elliptical trenches. Permalloy thin film was then thermally evaporated onto the wafer and the elements were transferred after dipping wafer into acetone. Four non-magnetic gold leads were patched on the element in the second step of electron beam lithography and a DC MR measurement was carried out with the external magnetic field applied along the short axis at room temperature. In addition, a magnetic force microscopy 
(MFM) was utilized for probing the magnetization configurations of the permalloy ellipses.

A typical transverse MR curve of sample A having three vortex-antivortex cores of cross-tie wall configuration is shown in Fig. 1(a). Notice that there are four steps developed in the MR curve at each quadrant. These steps are believed to be due to the annihilation or formation of vortex-antivortex core along the cross-tie wall. Figs. 1(b)-(e) are micromagnetic simulation showing magnetization evolution of a ellipse having two vortex-antivortex cores of cross-tie wall configuration and the corresponding MR curve is shown in Fig. 1(f), which was calculated by summing over the cross-section of the magnetization encountered by charge carriers during the magnetization evolution in the ellipse. The calculated MR feature has a somewhat similar behavior with the experimental data. The stepped MR behavior of sample A can be interpreted as follows. A vortex core followed by three vortex-antivortex cores was aligned to the film plane in sequence when the external magnetic field is increased, giving rise to a resistance drop. Consequently, four transitions may occur and so four steps developed in each quadrant of MR curve.

Fig. 2(a) is the transverse MR curve of sample B. The inset to the Fig. 2(a) is a MFM image showing a two-vortex configuration. This MR behavior can be explained with the schematics shown in Figs. 2(b)-(f). When the field starts decreasing from saturation field upward the central rhombus starts shrinking, therefore the resistance rises smoothly. Until the filed is kept decreasing to the opposite direction the resistance drops smoothly. However, at a critical negative field the central rhombic magnetization is diminished and rotated from parallel to the long axis direction, as shown in Fig. 2(e), the resistance then rises. The resistance decreases again when the field is kept
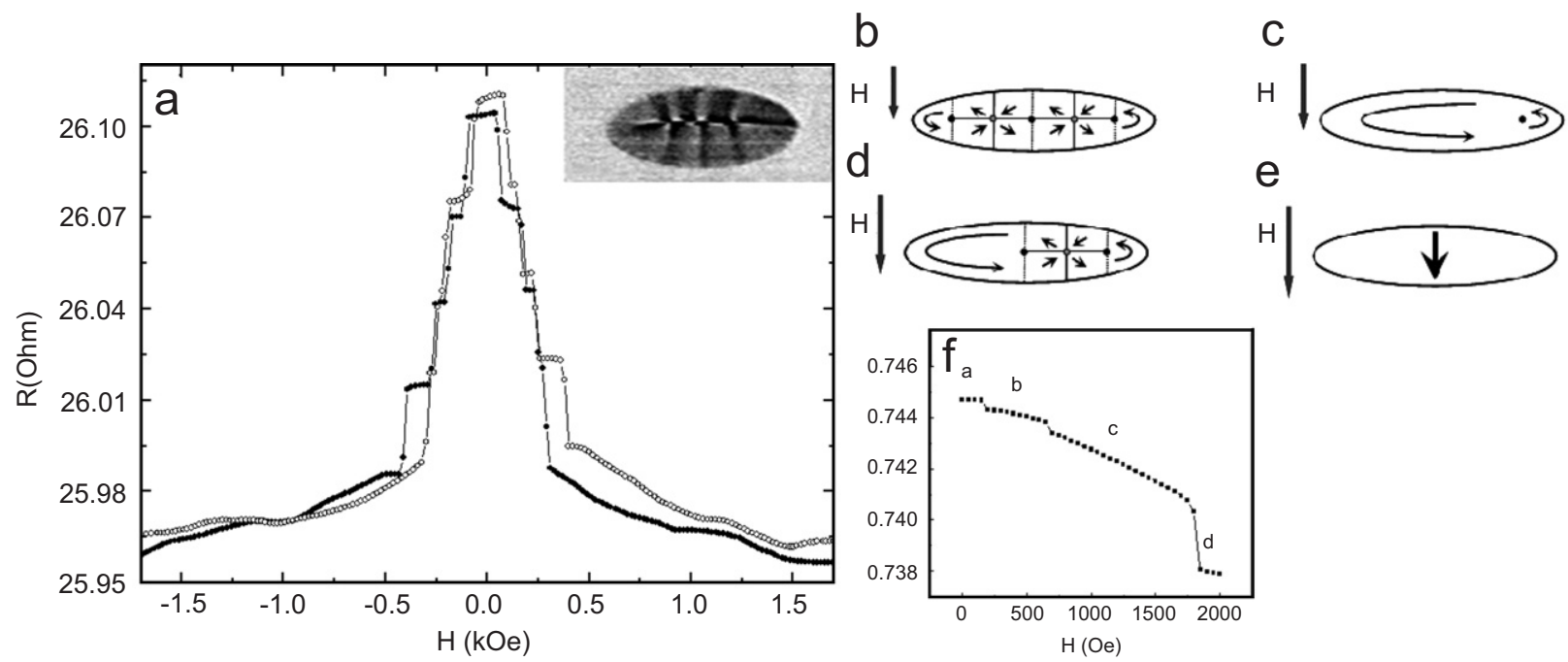

Fig. 1. (a) Transverse MR curves of sample A and the corresponding ellipse's MFM image. (b)-(e) Micromagnetic simulations of two cross-tie wall under different applied external fields. (f) The simulation MR curve of two cross-tie wall as He increase form 0 Oe. The long/short axes are $2 / 1 \mu \mathrm{m}$ and thickness is $40 \mathrm{~nm}$. Note that the origin of the MR steps can be attributed to the evolution of cross-tie walls.

a

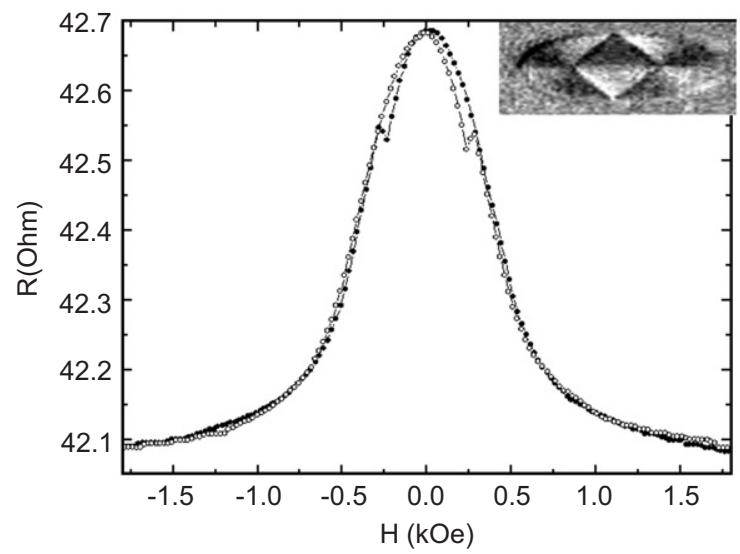

b

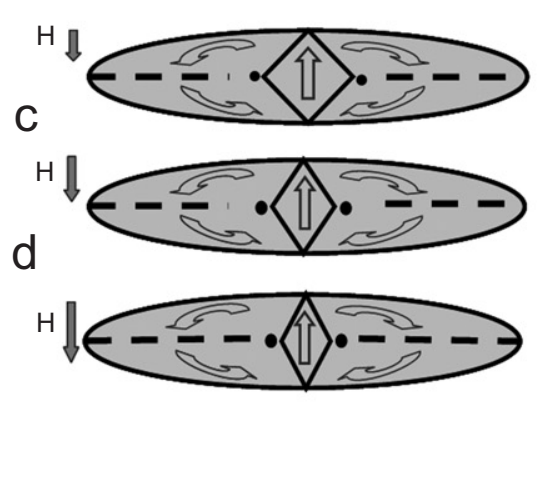

e

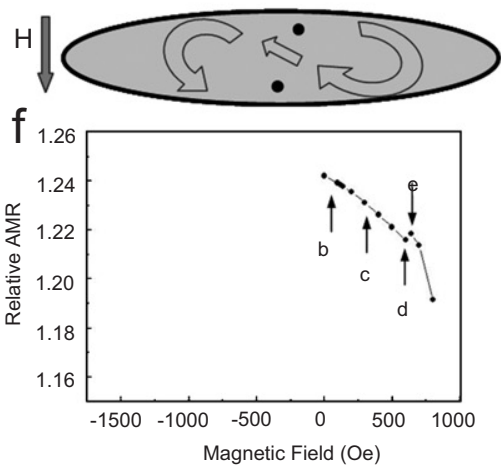

Fig. 2. (a) Transverse MR curve of sample B and the corresponding ellipse's MFM image. The long/short axes are $8 / 1 \mu \mathrm{m}$ and thickness is $55 \mathrm{~nm}$. (b)-(e) Micromagnetic simulations of two vortex under different external fields. (f) The simulation MR curve as $\mathrm{H}_{\mathrm{e}}$ increase form 0 Oe. Note that the origin of the MR kinks can be attributed to the evolution of vortex cores. 


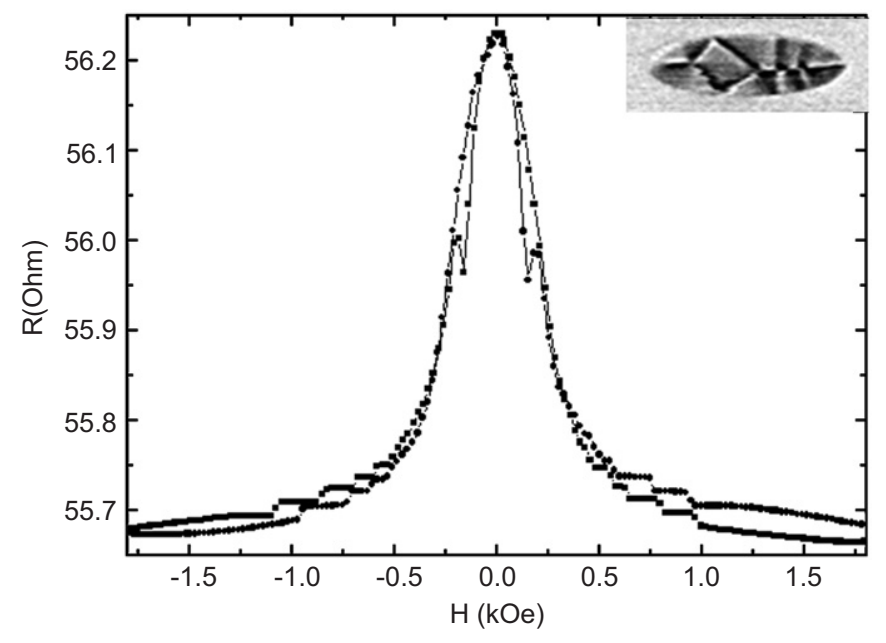

Fig. 3. Transverse MR curve of sample c and the coresponding ellipse's MFM image. The long/short axes are $5 / 1 \mu \mathrm{m}$ and thickness is $33 \mathrm{~nm}$. Note that the origin of the MR kinks and steps can be attributed to the evolution of combination of vortex cores and cross-tie walls.

decreasing to the negative direction. As a result, a kink occurs at the critical field.

Fig. 3 is the transverse MR curve of sample $\mathrm{C}$, which more or less shows a combination of previous two MR curves. The inset to the Fig. 3 is a MFM image showing vortex along with cross-tie wall configuration. It is, therefore, reasonable to argue that the MR behavior is combining the effects occurred on samples A and B.

In summary, we have presented a series of MR curves with characteristic behaviors. Three elliptical permalloy elements were purposely designed for forming certain magnetization configurations. Consequently, the one with cross-tie wall shows stepped MR behavior, the one with two-vortex configuration reveals kinked MR behavior, and the one with vortex and cross-tie wall configuration gives MR curve combining steps and kinks.

This work was supported by the Ministry of Economic Affairs of Taiwan with Grant no. 94-EC-17-A-08-S1-0006 and the National Science Council Grant with no. NSC 942112-M-018-002.

\section{References}

[1] R. Hunt, IEEE Trans. Magn. Mag 7 (1971) 150.

[2] C. H. Tsang, R. E. Fontana Jr, T. Lin, D. E. Heim, IBM J. Res. Develop. (Jan 1998).

[3] Gary A. Prinz, Science 282 (1998) 1660.

[4] J. Aumentado, V. Chandrasekhar, Appl. Phys. Lett. 74 (1999) 1898.

[5] P. Vavassori, M. Grimsditch, V. Metlushko, N. Zaluzec, B. Ilic, Appl. Phys. Lett. 86 (1999) 072507. 